Purdue University Purdue e-Pubs

2011

\title{
Assessment of Nanostructured Capillary Wicks for Passive, Two-Phase Heat Transport
}

\author{
R. Ranjan \\ Purdue University \\ S V. Garimella \\ Purdue University, sureshg@purdue.edu \\ J.Y. Murthy \\ Purdue University \\ K. Yazawa \\ Purdue University
}

Follow this and additional works at: http:// docs.lib.purdue.edu/coolingpubs

Ranjan, R.; Garimella, S V.; Murthy, J. Y.; and Yazawa, K., "Assessment of Nanostructured Capillary Wicks for Passive, Two-Phase Heat Transport" (2011). CTRC Research Publications. Paper 158.

http://dx.doi.org/10.1080/15567265.2011.597492

This document has been made available through Purdue e-Pubs, a service of the Purdue University Libraries. Please contact epubs@purdue.edu for additional information. 


\title{
Assessment of Nanostructured Capillary Wicks for Passive Two-Phase Heat Transport
}

\author{
Ram Ranjan ${ }^{1}$, Suresh V. Garimella ${ }^{1 \S}$, Jayathi Y. Murthy ${ }^{1}$, Kazuaki Yazawa ${ }^{2}$ \\ ${ }^{1}$ School of Mechanical Engineering and Birck Nanotechnology Center \\ Purdue University, West Lafayette IN 47907 USA \\ ${ }^{2}$ University of California at Santa Cruz, Santa Cruz CA 95064 USA
}

ranjanr@purdue.edu, sureshg@purdue.edu,jmurthy@purdue.edu, kaz@soe.ucsc.edu

\begin{abstract}
The major factors limiting the thermal performance of passive two-phase heat-spreading devices are the ability of the wick structures to transport liquid by means of capillary forces and the thermal resistance posed by the wicks. Nano-scale geometric enhancements to the wick structure, through the use of carbon nanotubes and metallic nanowires, promise to enhance the capillary transport while at the same time decreasing the thermal resistance due to their high intrinsic thermal conductivity. We analyze the performance of nanostructured wicks in heat-spreading applications. We report that the flow resistance of nanostructures constitutes a major barrier to
\end{abstract}

${ }^{\S}$ Author to whom correspondence should be addressed. Phone: +1 (765) 494-5621 
their use as passive flow-conveying media, and identify geometrical parameters which yield high rates of thin-film evaporation while minimizing the flow resistance. The analysis shows that the use of nanostructures as the sole wicking element in a two-phase thermal spreader restricts its footprint area to a size of $4 \mathrm{~cm}^{2}$ for heat flux inputs as low as $1 \mathrm{~W} / \mathrm{cm}^{2}$ due to the large flow resistance in the nanowick. To overcome nanowick flow resistance, we propose a nanostructureenhanced sintered particle wick microstructure which leads to a decrease in the wick thermal resistance by $14 \%$ relative to the corresponding wick with the same flow resistance and without nanostructures.

\section{INTRODUCTION}

Vapor chambers [1,2] (Figure 1(a)) and heat pipes are widely used as passive heat spreaders in industry. Because the working fluid undergoes phase change by evaporation or boiling, the effective thermal conductivity of vapor chambers is much higher than that of solid-block heat spreaders. In a typical arrangement, the working liquid undergoes phase change to vapor in the evaporator and is transported to the condenser where it condenses back into liquid. The requisite pressure gradient is provided by the capillary action of a wick structure, typically sintered copper particles, grooves, screen mesh, or a combination, which carries the condensed liquid from the condenser back to the evaporator, thus completing the flow loop. For an operational vapor chamber, the sum of the liquid and vapor pressure drops must be less than the available capillary pressure head of the wick structure between evaporator and condenser sections. One of the major limits to vapor chamber performance comes from the capillary limit [3] of the wick structure, which determines the maximum heat input that the device can sustain without the occurrence of dry-out. The performance is also adversely affected by the thermal resistance of the wick layer $[1,4,5]$. Heat is transferred through the saturated wick medium by conduction and at the liquid 
meniscus formed in the wick pores by evaporation. For macro-scale wicks, the thermal resistance of the wick medium tends to be the governing resistance to heat flow [6]. However, the recent development of ultra-thin nanostructured wicks may significantly reduce this resistance; in this limit, the evaporative resistance may begin to compete. The evaporative resistance scales directly as the liquid film thickness in the meniscus. The thin-film region [7-9] of a liquid meniscus has been defined in different ways, but generally represents a liquid film less than $1 \mu \mathrm{m}$ in thickness [10] near the solid-liquid contact line. Liquid molecules in the thin-film region experience intermolecular attractive forces with the solid, leading to a favorable pressure gradient for evaporation. Evaporation occurring from the thin-film region of the meniscus has been shown to contribute as much as $60-70 \%$ of the total heat transfer occurring from the meniscus [7,10-12]. Hence, wick structures that lead to a large thin-film area at a given heat load can decrease the overall wick thermal resistance by decreasing the liquid film thickness. Nanostructures offer the possibility of engineering wicks which can generate large capillary heads as well as support large thin-film areas.

Recently, Chen et al. [13] reported a $100 \%$ increase in the critical heat flux and heat transfer coefficient during boiling of high surface tension liquids in silicon and copper nanowire (NW) arrays. They attributed these increases to the superhydrophilicity of NWs, the large number of pores or cavities in the NW arrays, and their high wicking capability. Carbon nanotubes (CNTs) were suggested as efficient wick structures for vapor chambers by Vadakkan et al. [14]. They studied the wettability of CNT arrays with fluids such as FC-72, a perfluorinated dielectric liquid, and pointed out the benefits of dense CNT wicks over microstructured wicks. In another study, Ujereh et al. [15] performed pool boiling experiments on CNT-coated $\mathrm{Si}$ and $\mathrm{Cu}$ surfaces. They observed increases of $45 \%$ and $6 \%$, respectively, in the critical heat flux for fully 
CNT-coated $\mathrm{Si}$ and $\mathrm{Cu}$ surfaces. Nanowicks have been proposed as miniature wicking elements for liquid delivery and potential microfluidic chemical analysis devices. Zhou et al. [16] proposed nanowick strips composed of dense arrays of CNTs for applications such as microchromatography, rate control of micro-combustion, and microchip cooling.

Nanostructured wicks or "nanowicks" fabricated from CNTs and NWs are promising for the following reasons:

(1) Nanowicks have a significantly higher number of wick pores per unit substrate area than conventional wicks, thereby increasing the thin-film area available for evaporation, and potentially offering a significant increase in nucleation sites. Excellent boiling properties (higher CHF and heat transfer coefficient) have been reported for CNT- and NW-coated surfaces [13], making nanowicks ideal candidates for dissipating high heat fluxes.

(2) The nanoscale pores of wetting nanowicks can lead to high capillary pressure which may increase the capillary limit of vapor-chamber heat spreaders. The hydrophilicity of NWs and CNTs with water has been widely reported in the literature [13,17-19]. Sessile-drop experiments performed by Ebbesen [20] with water on a CNT bundle showed complete wetting of the capillary channels between nanotubes. Rossi et al. [19] observed the liquid menisci inside straight, CVD-fabricated CNTs using environmental scanning electron microscopy (ESEM). They reported a water-CNT contact angle varying between 5 and 20 degrees. Similar observations regarding the hydrophilicity of CNT surfaces were made by Kim et al. [21]. Hydrophilicity and wetting contact angles can be expected to support the generation of high capillary pressure. 
(3) CNTs have been reported to have a high thermal conductivity [22-26]. Wick thermal resistance, a significant bottleneck to vapor chamber performance, may be significantly reduced by such highly-conducting nanostructures.

However, while CNT and NW structures may offer high capillary pressures and large thin-film areas, a significant challenge in their use is their relative impermeability. The high liquid pressure drop that results hinders the establishment of continuous flow in the vapor chamber devices.

In this paper, we assess the feasibility of using nanowicks (carbon nanotubes and metallic nanowires grown on a smooth copper substrate) as wick materials in vapor chambers for thermal management. We determine optimal geometric arrangements of the CNTs/NWs necessary to obtain satisfactory thermal performance.

\section{MODELING APPROACH}

Three factors compete to determine whether nanowicks are viable for use in vapor chambers: capillarity, permeability, and wick thermal resistance. We develop theoretical and numerical models for these three parameters to analyze the performance of nanowicks, under the following assumptions:

(1) CNTs are idealized as vertically aligned posts in both hexagonal- (Figure 1(b)) and squarepacked (Figure 1(c)) arrangements on the substrate. Figure 1(d) shows the plan view of the square-packed posts, and $p$ denotes the pitch non-dimensionalized by the diameter of the CNT. It should be noted that CNT arrays are characterized by varying pore sizes and packing arrangements. However, the present analysis based on an idealized arrangement of CNT pillars 
holds for arbitrarily-grown CNT forests which have the same spatially averaged pore radius as the idealized case, at least in terms of the essential trends of behavior.

(2) The contact angle $(\theta)$ of water with the CNT surface is assumed to be $15^{\circ}$, based on the range of contact angles observed by Rossi et al. [19] and Kim et al. [21].

(3) Continuum approximations are made in determining the liquid meniscus shape in the wick pore and the liquid flow and heat transfer at the scale of CNTs and NWs (diameter range $=40$ $\mathrm{nm}-300 \mathrm{~nm}$ ). Kim et al. [21] compared their experiments on capillary filling and the evaporation and condensation of water in CNTs (diameter range $=300-800 \mathrm{~nm}$ ) with simple models based on continuum theories and found good agreement. Moreover, the hydrodynamics of water passing through CNT arrays has been studied numerically by Walther et al. [27] and the predicted drag coefficients were found to be in reasonable agreement with macroscopic theory. In other experiments, Fradin et al. [28] concluded that the surface tension of water with its vapor remains unchanged down to a length scale of $10 \mathrm{~nm}$. These studies support the choice of a continuum approach in our work.

The number density $\Phi$ and the diameter $D$ of the CNTs or NWs are varied in the present study to obtain optimal parameters for the best performance of the nanowick. The quantities $\Phi$, $D$, wick porosity $\varepsilon$, pitch $p$ (Figure $1(\mathrm{~d})$ ) and base area of the unit cell $A_{b}$ are related to each other for the hexagonal and square-packed arrangements in the manner shown in Table 1.

2.1 Nanowick Capillarity Model. The capillary pressure $\Delta P_{\text {cap }}$ generated by a wick depends on the mean curvature of the liquid meniscus $H$ formed in its pore and the surface tension $\gamma_{L V}$ of the liquid, as given by the Young-Laplace equation [29]:

$$
\Delta P_{c a p}=\gamma_{L V}\left(\frac{1}{r_{1}}+\frac{1}{r_{2}}\right)=2 H \gamma_{L V}
$$


We obtain the mean curvature of the liquid meniscus in a nanowick pore under prescribed constraints (liquid volume, contact angle) using the surface energy minimization program, SURFACE EVOLVER [30,6]. The meniscus shape depends on the geometry (CNT diameter, wick porosity) and the solid-liquid contact angle. The CNT diameter and wick density are varied over 40-300 nm and $10^{6}-10^{10}$ per $\mathrm{cm}^{2}$, respectively, to study the effect on $\Delta P_{\text {cap }}$. These values are in keeping with values obtained in typical fabrication processes [31-33]. Figure 2(a) shows the shape of the water meniscus in a nanopore formed in the case of square-packed posts for given geometrical parameters $\left(p=2, h_{m}=r\right) ; h_{m}$ is the height of the liquid meniscus in the nanopore.

2.2 Nanowick Permeability Model. Since established correlations for the permeability of short nanowicks on a substrate are not available in the literature we model liquid flow across nanowicks using FLUENT ${ }^{\circledR}$, a finite-volume based flow solver [34]. Low-Reynolds number flow with $R e=0.1$ (Reynolds number, $R e=\rho u D / \mu ; u$ is liquid velocity, $\mu$ and $\rho$ are liquid dynamic viscosity and density, respectively) is modeled for both hexagonally- and square-packed vertical posts. A two-dimensional model with a unit-cell representation of CNTs is developed. A periodic flow boundary condition is used at the liquid inlet and outlet boundaries. Computed contours of the pressure $P$ around a CNT are shown in Figure 2(b). The permeability $(K)$ is obtained using Darcy's law [35]

$$
\nabla P=-\frac{\mu \dot{m}}{K A \rho}
$$

in which $A$ is the flow cross-sectional area and $\dot{m}$ is the liquid mass flow rate.

2.3 Wicking Length along Nanowick. The vapor pressure drop in the chamber may be assumed to be insignificant compared to the liquid pressure drop in the wick. The capillary pressure head 
generated by the wick structure must be greater than the liquid pressure drop in the wick in order to prevent dry-out. The wicking length is a useful parameter with which to assess the performance of nanowicks. We define it as the length to which a given mass flow rate can be transported by the action of wick capillary pressure. It is obtained by equating the wick capillary pressure with the liquid pressure drop for a given liquid mass flow rate, in the configuration illustrated in Figure 2(d). For a given input heat flux, $q^{\prime \prime}$, the incoming liquid mass flow rate $\dot{m}$ per unit width of the nanowick is represented as:

$$
\dot{m}^{\prime}=\frac{\dot{q}^{\prime \prime}}{h_{f g} L_{w}}
$$

The liquid mass flow rate per unit width of the nanowick can also be represented in terms of the flow pressure drop using Eq. (2), as follows:

$$
\dot{m}^{\prime}=\frac{K L_{C N T} \rho \Delta P}{\mu L_{w}}
$$

The wicking length $\left(L_{w}\right)$ can then be written as a function of wick thickness (or CNT height $\left.L_{C N T}\right), \Delta P_{c a p}$ and $K$ as

$$
L_{w}=\sqrt{\frac{K L_{C N T} \rho \Delta P_{c a p} h_{f g}}{\mu \dot{q}^{\prime}}}
$$

in which $h_{f g}$ is the latent heat of vaporization of water. Eq. (5) is obtained by equating the mass flow rates in Eqs. (3) and (4) with $\Delta P=\Delta P_{\text {cap }}$.

Higher wicking lengths are desired for designing vapor chambers which resist dry-out.

2.4 Evaporation Model. The evaporative resistance of the liquid meniscus formed in the nanopore is computed numerically using an evaporating meniscus model [36] developed previously. Evaporation at the liquid-vapor interface in the nanopore is computed for a given 
wall superheat under saturated vapor conditions using the relation given by Schrage [37]. Temperature contours in the liquid and solid domains for one such case are shown in Figure 2(c). The evaporative heat transfer coefficient $\left(h_{\text {evap }}\right)$ at the liquid-vapor interface is obtained using these simulations.

2.5 Thermal Resistance Model of Nanowick. The thermal resistance of the nanowick is estimated using the resistance network model shown in Figure 3(a) and (b). The thermal resistance from the substrate to the vapor consists of three resistances, viz., the conduction resistance through the liquid $\left(R_{1}\right.$ and $\left.R_{2}\right)$ and the evaporative resistance at the liquid meniscus, $R_{3} . R_{1}$ depends on the shortest path for heat conduction from the CNT wall to the meniscus while $R_{2}$ depends upon the CNT height, $h_{m}$. For calculating $R_{3}$, an evaporative heat transfer coefficient

of $8.7 \times 10^{5} \mathrm{~W} / \mathrm{m}^{2} \mathrm{~K}$ at the liquid meniscus is obtained from the evaporation model discussed above. The area-averaged shortest distance of the meniscus from the CNT wall and the meniscus area are both obtained from the capillarity model.

We now discuss results obtained from the various models presented above.

\section{RESULTS AND DISCUSSION}

3.1 Capillary Pressure and Permeability of Nanowick. The variation of $\Delta P_{c a p}$ and $K$ of the nanowick are studied as functions of the wick density, porosity and CNT diameter. Figure 4(a) shows the variation of $\Delta P_{c a p}$ with $\Phi$ and $D$ for both hexagonal- and square-packed arrangements. The capillary pressure increases with increasing CNT density $\Phi$. When the number of CNTs per unit substrate area increases, the wick pore size decreases, leading to an increase in $\Delta P_{c a p}$. For a 
given $\Phi$, the wick porosity depends on the CNT diameter. A larger diameter leads to smaller porosity (Table 1), again leading to an increase in $\Delta P_{c a p}$. For $\Phi>10^{9}$ per $\mathrm{cm}^{2}, \Delta P_{c a p}$ (which is > $10^{5} \mathrm{~Pa}$ ) is 2-3 orders higher than that of a typical sintered particle wick which is of order of $10^{3}$ Pa. The permeability $K$ is normalized with $r^{2}$ and its variation with wick porosity for both packing arrangements is plotted in Figure 4(b). $K$ increases with increasing wick porosity $\varepsilon$. Alternatively, $K$ decreases with increasing nanowick density $\Phi . D$ and $\varepsilon$ are varied from 100$1000 \mathrm{~nm}$ and $0.3-0.99$, respectively to obtain permeability correlations for different porosity ranges using a least-squares curve fit as follows:

$$
\begin{gathered}
K=\frac{0.0722 r^{2} \varepsilon^{4.68}}{(1-\varepsilon)^{1.62}} \quad \text { Hexagonal packing } \\
K=\frac{0.4191 r^{2} \varepsilon^{5.48}}{(1-\varepsilon)^{1.1}} \quad \text { Square packing }
\end{gathered}
$$

3.2 Wicking Length and Thermal Resistance of Nanowick. An input heat flux $\left(q^{\prime \prime}\right)$ of $1 \mathrm{~W} / \mathrm{cm}^{2}$ and $L_{C N T}$ of $100 \mu \mathrm{m}$ are assumed in obtaining the wicking length along the nanowick. As shown in Figure 4, the wicking length first increases and then decreases with increasing nanowick density $\Phi$. Both the available capillary head $\left(\Delta P_{c a p}\right)$ and the liquid flow resistance (i.e., the viscous pressure drop determined by $K$ ) increase with increasing $\Phi$. From Eq. (3), $L_{w}^{2}$ is proportional to $K \Delta P_{c a p}$. The product $K \Delta P_{c a p}$ therefore first increases and then decreases with increasing wick density. The trade-off between $\Delta P_{c a p}$ and $K$ leads to a maximum in $L_{w}$ at a nondimensional pitch $p \sim 5$ (this pitch corresponds to the peaks observed in Figure 4(c) and (d) for various CNT diameters) for all cases of CNT diameters in both packing arrangements. The optimum pitch is determined to be approximately $5 D$ for any CNT diameter. Also, wicking lengths are very small $\left(\left.L_{w}\right|_{\max }=2 \mathrm{~cm}\right.$ for $\left.D=300 \mathrm{~nm}\right)$ even for a modest input heat flux of 1 
$\mathrm{W} / \mathrm{cm}^{2}$. For higher heat fluxes, $L_{w}$ would be even smaller. This suggests that nanowicks on a smooth substrate would perform poorly in the evaporator section of typical heat spreaders for microelectronics thermal management, which have footprint areas larger than $4 \mathrm{~cm}^{2}$. The primary shortcoming of nanowicks is the liquid flow resistance.

The variation of thermal resistance with $\Phi$ and $D$ is shown in Figure 5(a) (hexagonal packing) and (b) (square packing). The wick thermal resistance depends on the liquid level $h_{m}$ in the wick pore, which is varied to study its effect on thermal resistance. It is observed that the thermal resistance of the nanowick (of order of $1 \mathrm{Kmm}^{2} / \mathrm{W}$ ) is much smaller than the wick resistance of typical sintered or screen-mesh wicks which is of order $1 \mathrm{Kcm}^{2} / \mathrm{W}$ [3-6]. Also, the wick resistance becomes independent of the CNT density, diameter and liquid level for large densities $\left(>10^{8}\right.$ per $\mathrm{cm}^{2}$ ). The wick resistance calculation does not account for the CNT-Cu interface thermal resistance. Cola et al. [38,39] reported this interface resistance to be of the order of $1 \mathrm{Kmm}^{2} / \mathrm{W}$ for moderate applied pressures. Therefore, the interfacial thermal resistance of nanowicks would be equivalent to the conduction resistance of the liquid and the evaporative resistance of the meniscus combined. Even with this added interface resistance, it is clear that nanowicks would significantly outperform existing commercially available wicking materials in terms of thermal resistance.

3.3 Nanostructure-enhanced Microstructured Wicks. As discussed above, nanowicks have excellent thermal properties for use in vapor chambers. However, the capillary head generated by the nanopores is not sufficient to support liquid flow over a distance greater than $2 \mathrm{~cm}$. To overcome the difficulties posed by the low nanowick permeability, we propose an alternative wick design with high permeability and enhanced evaporation characteristics. We now study a 
case where the surface of a sintered copper particle wick structure with high permeability $(K \sim$ $10^{-10} \mathrm{~m}^{2}$ ) [3] is enhanced using CNTs or NWs. Such a design for the wick structure is inspired by the use of bi-porous wick structures [40] and integrated nanostructured wicking surfaces [41] for enhancing wick permeability and reducing its thermal resistance. Heat pipes made with sintered particle wick structures have been shown [42] to sustain high heat fluxes of up to $100 \mathrm{~W} / \mathrm{cm}^{2}$. Moreover, theoretical models [6] have shown that sintered particle wick structures possess the highest wicking capability among common wick structures and provide enhanced thin-film evaporation. If CNTs are grown on the particles in the wick, the number of wick pores for evaporation heat transfer would be enhanced. In this work, we utilize simple theoretical and numerical models to estimate the maximum and minimum gains in thermal performance of sintered particle wick structures due to nanostructuring using CNTs.

An increase in the thin-film area due to the CNTs present on the sintered particles is estimated using the capillarity model. The meniscus area in a given particle arrangement and solid-liquid contact angle is again computed using the program SURFACE EVOLVER. Sintered particles are assumed to have a square-packed arrangement with $\varepsilon=0.56$ and particle diameter $=$ $100 \mu \mathrm{m}$ (Figure 6(a)). The CNTs are assumed to be square-packed vertical posts on the surface of the particles. The CNT density $\Phi$ is varied from $10^{6}$ to $4 \times 10^{10}$ per $\mathrm{cm}^{2}$ with a CNT diameter $D=$ $50 \mathrm{~nm}$ and a CNT height $L_{C N T}=1 \mu \mathrm{m}$. The area of the liquid meniscus covering the particle is computed using the capillarity model, but only a film thickness smaller than $L_{C N T}(1 \mu \mathrm{m})$ is considered since only CNT pores that are not fully submerged contribute to the enhancement of the thin-film area. The number of such CNT pores is computed based on the given CNT density. For each CNT pore, the meniscus area is computed using the nanowick capillarity model, assuming a CNT-water contact angle of $15^{\circ}[19,21]$. The number of CNT pores which contribute 
to the enhancement of the thin-film area depends on the wicking ability of the CNT forest. We study two extreme cases: (1) when CNTs are non-wicking (Figure 6(b)), and (2) when the CNTs are completely wicking (Figure 6(c)) and all CNT pores on the particle surface contribute to enhancement in thin-film area.

The thermal resistance of the CNT-enhanced sintered particle wick is computed using a resistance network model, shown in Figure 7(a) \& (b). This is an approximate resistance model which accounts for conduction and evaporation through the wick structure. This model does not apply when estimating the wick thermal resistance at high heat inputs (when boiling occurs in the wick structure) and/or when there is high contact resistance between the particles of the wick structure. A similar model was used by the authors [6] to estimate the thermal resistance of sintered particle wicks. An octahedral volume around a spherical particle in the wick is chosen as the representative unit cell. A three-dimensional packing of spheres with $N$ layers is assumed. The particles are hexagonally packed in each layer and arranged in a simple cubic packing in the vertical direction. Heat flows from the hot substrate wall to the liquid-vapor interface by conduction. Convection in the liquid may be ignored in comparison to conduction through the solid sintered material. Also, thermocapillary convection is not considered because its effect on heat transfer has been shown to be negligible at small superheats [36]. Heat transfer from the liquid to vapor occurs by evaporation at the liquid-vapor interface formed in the top layer of the wick pores. The temperature varies along the liquid meniscus because of the variation in evaporation rates resulting from the variation in film thickness [36]. Hence, two separate paths are considered, viz., the thin-film path and the intrinsic meniscus path (or the non-thin film path), for heat transfer from the top layer of spheres to the vapor [6]. A separate heat transfer path through the meniscus formed in the nanopores of the CNTs is included in the network. The seven 
thermal resistances $R_{1}, R_{2}, R_{3}, R_{4}, R_{5}, R_{6}$ and $R_{7}$, shown in Figure 7(a), result from: (i) $R_{1}$ : the conduction resistance due to (N-1) layers of spheres (solid) and interstitial liquid, (ii) $R_{2}, R_{3}, R_{6}$ : the thermal resistance offered by the liquid for conduction from the top layer of spheres to the menisci formed in the thin-film region, the intrinsic region and the CNT pores, respectively, and (iii) $R_{5}, R_{4}, R_{7}$ : the evaporation resistance of the liquid menisci formed in the thin-film region, the intrinsic region and the CNT pores, respectively. These resistances can be represented in terms of the meniscus area, the thin-film area and the shortest path to the meniscus from the sphere surface (for conduction resistances), computed from the capillarity models [6]. The total wick thermal resistance due to conduction in the liquid and evaporation at the liquid-vapor interface (network shown in Figure 7(b)) is computed for varying CNT density. We note that the enhancement in thin-film area will depend on the number of CNT pores. The maximum number of such CNT pores depends on the un-submerged surface area of the particles, and is determined by the meniscus level $h$ in the wick pore (Figure 7(a)). We vary the meniscus level $h$ to determine its effect on the total wick resistance.

The evaporation heat transfer coefficients at the liquid menisci formed in the particle and CNT pores are computed using the evaporation model [36] as discussed before. The particle (copper)-water contact angle is assumed to be $30^{\circ}$ [3] while the CNT-water contact angle is taken as $15^{\circ}[19,21]$. The effective thermal conductivity of the saturated sintered particle wick determines the thermal resistance due to underlying particle layers $\left(R_{l}\right)$ in the sintered wick and is assumed to be $5 \mathrm{~W} / \mathrm{mK}[3,43]$.

The enhancement of a particle surface with CNTs leads to a decrease in the wick thermal resistance, as shown in Figure 8. The minimum wick resistance is realized for the case when $h / r_{p}$ $=0.6$ and the CNTs are completely wicking. For $h / r_{p}=0.6$, the number of CNT pores on the 
particle surface is larger than those for $h / r_{p}=1$ and 1.4. It can be concluded that growing CNTs on the particle surface will lead to a decrease in the thermal resistance of the wick for all cases (wetting/non-wetting CNTs) except when $\Phi>3 \times 10^{10}$ per $\mathrm{cm}^{2}$ (corresponding to $p<1.15$ ). For very high CNT densities $\left(\Phi>10^{10}\right.$ per $\left.\mathrm{cm}^{2}\right)$, the thermal resistance of the CNT-enhanced wick starts to increase mildly. This is because an increase in CNT density leads to a larger number of nanopores on the particle surface. It is accompanied by a decrease in the meniscus area formed in each nanopore. The total area of the liquid menisci formed in the CNT nanopores is found to first increase with increasing $\Phi$ and subsequently decreases when $\Phi>10^{10}$ per $\mathrm{cm}^{2}$. This leads to a mild initial decrease in the total wick thermal resistance as the CNT density is increased. The minimum thermal resistance is observed at a CNT density $=10^{9}$ per $\mathrm{cm}^{2}$. The maximum decrease in wick thermal resistance $\left(h / r_{p}=0.6, \Phi=10^{9}\right.$ per $\mathrm{cm}^{2}$ for completely wicking CNTs) is $14 \%$ as compared to the no-CNT case (Figure 6(a)). For the case of non-wicking CNTs, the maximum decrease in thermal resistance due to CNTs is approximately 3\%. Completely non-wicking CNTs lead to an increase in the wick thermal resistance for $\Phi=4 \times 10^{10}$ per $\mathrm{cm}^{2}$. From this study, we conclude that hydrophilic CNTs of moderate densities $\left(\Phi=10^{8}-10^{10}\right.$ per $\left.\mathrm{cm}^{2}\right)$ on sintered particles can decrease the thermal resistance of the wick structure by 3 - $14 \%$ depending upon the wicking ability of the CNTs. The extent of wicking in the CNTs determines the enhancement in thermal performance of the wick structure. Our earlier analysis shows that hydrophilic CNTs will be able to wick the liquid all along the exposed particle surface by capillary action since the required $L_{w}$ in this case is only $0.2 \mathrm{~mm}$.

\section{CONCLUSIONS}


In the present work, we have assessed the feasibility of using nanowicks (carbon nanotubes and metallic nanowires grown on a planar copper substrate) as wick materials in heat spreading devices such as vapor chambers for passive fluidic transport and thermal management. We have developed models based on continuum analyses to predict capillarity, permeability and thermal resistance of nanowicks. Nanowicks, due to their small dimensions, can generate much higher capillary pressure than typical commercially available wicks. However, the permeability of these wicks is very small, leading to very high resistances to liquid flow. An optimum pitch (center-to-center distance between CNTs) of approximately five times the post diameter can achieve the maximum wicking length in nanowicks. Our analysis shows that the use of nanowicks can lead to a decrease in the wick thermal resistance by 2 orders of magnitude compared to commercially available wicks. To overcome the limitations due to low permeability, we propose a CNT-enhanced sintered particle wick microstructure and show that hydrophilic CNTs can lead to a $14 \%$ decrease in the thermal resistance of the wick.

\section{REFERENCES}

[1] M. Lu, L. Mok and R. J. Bezama, A Graphite Foams Based Vapor Chamber for Chip Heat Spreading, Journal of Electronic Packaging, vol. 128, pp. 427, 2006.

[2] I. Sauciuc, G. Chrysler, R. Mahajan and R. Prasher, Spreading in the Heat Sink Base: Phase Change Systems or Solid Metals?, IEEE Transactions on Components and Packaging Technologies, vol. 25(4), pp. 621-628, 2002.

[3] A. Faghri, Heat Pipe Science and Technology, Taylor \& Francis, Washington, DC, 1995. 
[4] B. D. Iverson, T. W. Davis, S. V. Garimella, M. T. North and S. S. Kang, Heat and Mass Transport in Heat Pipe Wick Structures, Journal of Thermophysics and Heat Transfer, vol. 21(2), pp. 392-404, 2007.

[5] T. W. Davis and S. V. Garimella, Thermal Resistance Measurement across a Wick Structure using a Novel Thermosyphon Test Chamber, Experimental Heat Transfer, vol. 21, pp. 143-154, 2008.

[6] R. Ranjan, J. Y. Murthy and S. V. Garimella, Analysis of the Wicking and Thin-film Evaporation Characteristics of Wick Microstructures, ASME Journal of Heat Transfer, vol. 131, pp. 101001, 2009.

[7] M. Potash and P. C. Wayner, Evaporation from a 2-Dimensional Extended Meniscus, International Journal of Heat and Mass Transfer, vol. 15(10), pp. 1851-1863, 1972.

[8] X. Xu and V. P. Carey, Film Evaporation from a Micro-grooved Surface - An Approximate Heat Transfer Model and its Comparison with Experimental Data, Journal of Thermophysics and Heat Transfer, vol. 4, pp. 512-520, 1990.

[9] H. H. Sait and H. B. Ma, An Experimental Investigation of Thin-film Evaporation, Nanoscale and Microscale Thermophysical Engineering, vol. 13, pp. 218-227, 2009.

[10] H. Wang, S. V. Garimella and J. Y. Murthy, Characteristics of an Evaporating Thin Film in a Microchannel, International Journal of Heat and Mass Transfer, vol. 50, pp. 3933-3942, 2007. [11] P. C. Wayner Jr., Intermolecular Forces in change of Phase Heat Transfer: 1998 Donald Q. Kern Award Review, AIChE Journal, vol. 45(10), pp. 2055-2068, 1999.

[12] H. K. Dhavaleswarapu, C. P. Migliaccio, S. V. Garimella and J. Y. Murthy, Experimental Investigation of Evaporation from Low-Contact-Angle Sessile Droplets, Langmuir, vol. 26(2), pp. 880-888, 2009. 
[13] R. Chen, M. Lu, V. Srinivasan, Z. Wang, H. H. Cho and A. Majumdar, Nanowires for Enhanced Boiling Heat Transfer, Nanoletters, vol. 9(2), pp. 548-553, 2009.

[14] U. Vadakkan, G. M. Chrysler, J. Maveety and M. Tirumala, A Novel Carbon Nano Tube based Wick Structure for Heat Pipes/Vapor Chambers, Twenty-Third Annual IEEE Semiconductor Thermal Measurement and Management Symposium, SEMI-THERM Proceedings, pp. 102-104, 2007.

[15] S. Ujereh, T. Fisher and I. Mudawar, Effects of Carbon Nanotubes Arrays on Nucleate Pool Boiling, International Journal of Heat and Mass Transfer, vol. 50, pp. 4023-4038, 2007.

[16] J. J. Zhou, F. Noca and M. Gharib, Flow Conveying and Diagnosis with Carbon Nanotube Arrays, Nanotechnology, vol. 17, pp. 4845-4853, 2006.

[17] Y. Gogotsi, J. A. Libera, A. Guvenc-Yazicioglu and C. M. Megaridis, In situ Multiphase Fuid Experiments in Hydrothermal Carbon Nanotubes, Applied Physics Letters, vol. 79(7), pp. 1021-1023, 2001.

[18] J. -G. Fan, D. Dyer, G. Zhang and Y. -P. Zhao, Nanocarpet Effect: Pattern Formation during the Wetting of Vertically Aligned Nanorod Arrays, Nanoletters, vol. 4(11), pp. 2133-2138, 2004. [19] M. P. Rossi, H. Ye, Y. Gogotsi, S. Babu, P. Ndungu and J. -C. Bradley, Environmental Scanning Electron Microscopy Study of Water in Carbon Nanopipes, Nanoletters, vol. 4(5), pp. 989-993, 2004.

[20] T. W. Ebbesen, Carbon Nanotubes, Annual Review of Material Science, vol. 24, pp. 235, 1994.

[21] B. M. Kim, S. Sinha and H. H. Bau, Optical Microscope Study of Liquid Transport in Carbon Nanotubes, Nanoletters, vol. 4(11), pp. 2203-2208, 2004. 
[22] S. Berber, Y-K. Kwon and D. Tomanek, Unusually High Thermal Conductivity of Carbon Nanotubes, Physical Review Letters, vol. 84(20), pp. 4613, 2000.

[23] J. Che, T. Cagin and W. A. Goddard III, Thermal Conductivity of Carbon Nanotubes, Nanotechnology, vol. 11, pp. 65-69, 2000.

[24] P. Kim, L. Shi, A. Majumdar and P. L. McEuen, Thermal Transport Measurements of Individual Multiwalled Nanotubes, Physical Review Letters, vol. 87(21), pp. 215502, 2001.

[25] M. Fujii, X. Zhang, H. Xie, H. Ago, K. Takahashi, T. Ikuta, H. Abe and T. Shimizu, Measuring the Thermal Conductivity of a Single Carbon Nanotube, Physical Review Letters, vol. 95, pp. 065502, 2005.

[26] E. Pop, D. Mann, Q. Qang, K. Goodson and H. Dai, Thermal Conductance of an Individual Single-Wall Carbon Nanotube above Room Temperature, Nanoletters, vol. 6(1), pp. 96-100, 2006.

[27] J. H. Walther, T. Werder, R. L. Jaffe and P. Koumoutsakos, Hydrodynamic Properties of Carbon Nanotubes, Physical Review E, vol. 69, pp. 062201, 2004.

[28] C. Fradin, A. Braslau, D. Luzet, D. Smilgies, M. Alba, N. Boudet, K. Mecke and J. Daillant, Reduction in the Surface Energy of Liquid Interfaces at Short Length Scales, Nature, vol. 403, pp. 871-874, 2000.

[29] R. Finn, Capillary Surface Interfaces, Notices of the American Mathematical Society, vol. 46(7), pp. 770-781, 1999.

[30] K. A. Brakke, The Surface Evolver, Experimental Mathematics, vol. 1(2), pp. 141-165, 1992. 
[31] Z. P. Huang, J. W. Xu, Z. F. Ren, J. H. Wang, M. P. Siegal and P. N. Provencio, Growth of Highly Oriented Carbon Nanotubes by Plasma-enhanced Hot Filament Chemical Vapor Deposition, Applied Physics Letters, vol. 73(26), pp. 3845-3847, 1998.

[32] J. Li, C. Papadopoulos and J. M. Xu, Highly-ordered Carbon Nanotube Arrays for Electronics Applications, Applied Physics Letters, vol. 75(3), pp. 367-369, 1999.

[33] Y. Tu, Z. P. Huang, D. Z. Wang, J. G. Wen and Z. F. Ren, Growth of Aligned Carbon Nanotubes with Controlled Site Density, Applied Physics Letters, vol. 80(21), pp. 4018-4020, 2002.

[34] Fluent Inc., Fluent 6.2 User's Guide, 2004.

[35] S. Whitaker, Flow in porous media I: A theoretical derivation of Darcy's law, Transport in Porous Media, vol. 1(1), pp. 3-25, 1986.

[36] R. Ranjan, J. Y. Murthy and S. V. Garimella, A Microscale Model for Thin-film Evaporation in Capillary Wick Structures, International Journal of Heat and Mass Transfer, vol. 54 (1-3), pp. 169-179, 2011.

[37] R. W. Schrage, A Theoretical Study of Interface Mass Transfer, New York: Columbia University Press, 1953.

[38] B. A. Cola, J. Xu, C. Cheng, X. Xu, T. S. Fisher and H. Hu, Photoacoustic Characterization of Carbon Nanotube Array Thermal Interfaces, Journal of Applied Physics, vol. 101, pp. 054313, 2007.

[39] B. A. Cola, J. Xu and T. S. Fisher, Contact Mechanics and Thermal Conductance of Carbon Nanotubes Array Interfaces, International Journal of Heat and Mass Transfer, vol. 52, pp. 34903503, 2009. 
[40] T. Semenic and I. Catton, Experimental Study of Biporous Wicks for High Heat Flux Applications, International Journal of Heat and Mass Transfer, vol. 52 (21-22), pp. 5113-5121, 2009.

[41] J. A. Weibel, S. V. Garimella, J. Y. Murthy and D. H. Altman, Design of Integrated Nanostructured Wicks for High-Performance Vapor Chambers, IEEE Transactions on Components and Packaging Technologies, in press, 2011.

[42] N. J. Gernert, J. Toth and J. Hartenstine, $100 \mathrm{~W} / \mathrm{cm}^{2}$ and Higher Heat Flux Dissipation using Heat Pipes, $13^{\text {th }}$ International Heat Pipe Conference (IHPC), Shanghai, China, September 21-25, 2004.

[43] U. Vadakkan, S. V. Garimella and J. Y. Murthy, Transport in Flat Heat Pipes at High Fluxes from Multiple Discrete Sources, ASME Journal of Heat Transfer, vol. 126, pp. 347-354, 2004. 


\section{NOMENCLATURE}

A flow cross-sectional area

$A_{b} \quad$ base area of nanowick unit cell

D diameter of CNT/NW

$h_{\text {evap }} \quad$ evaporative heat transfer coefficient,

$h_{f g} \quad$ latent heat of vaporization

$h_{m} \quad$ height of the liquid meniscus in wick pore

$H \quad$ mean curvature of the liquid meniscus formed in nanowick

$K \quad$ wick permeability

$L_{C N T} \quad$ CNT height

$L_{w} \quad$ wicking length

$\dot{m} \quad$ liquid mass flow rate

$p \quad$ non-dimensional pitch (center-to-center distance between nanowires/CNTs)

$P \quad$ hydrodynamic pressure

$\Delta P_{\text {cap }} \quad$ capillary pressure

$q^{\prime \prime} \quad$ input heat flux

$r \quad$ radius

$r_{p} \quad$ particle radius

$r_{1}, r_{2} \quad$ radii of curvature

$R \quad$ thermal resistance

Re Reynolds number

$u \quad$ liquid velocity 


\section{Greek Symbols}

$\varepsilon$ nanowick porosity

$\gamma_{L V} \quad$ liquid-vapor surface tension

$\mu \quad$ liquid dynamic viscosity

$\Phi \quad$ CNT number density

$\rho \quad$ liquid density

$\theta \quad$ solid-liquid contact angle 


\section{LIST OF TABLES}

Table 1. Wick porosity, pitch and base area of unit cell in terms of CNT number density (per $\left.\mathrm{cm}^{2}\right)$ and diameter $(D)$.

\section{LIST OF FIGURES}

Figure 1. (a) Schematic diagram of the operation of a vapor chamber heat spreader. CNTs/NWs are shown in the evaporator region. $\mathrm{CNTs} / \mathrm{NWs}$ are shown as vertical posts in (b) hexagonal and (c) square packed arrangements on a copper substrate. (d) Plan view of square-packed posts with $D$ being the post diameter and $p$ the non-dimensional pitch.

Figure 2. (a) The shape of the water meniscus in the nanopore formed between square-packed CNTs. The meniscus surface is puckered, exhibiting anticlastic curvature in the narrower spaces between two adjacent posts, while the surface is synclastic in the central pore region. (b) Pressure contours (Pa) are shown in the liquid flow region around a square-packed post $(D=100 \mathrm{~nm}, p=1.4)$. The permeability of the nanowick is computed using Darcy's flow model for porous media. (c) Temperature contours $(\mathrm{K})$ during evaporation of water from the meniscus formed in a nanopore (square-packed posts) under saturated vapor conditions (wall superheat $=2.5 \mathrm{~K}, \theta=$ $15^{\circ}, T_{\text {vap }}=298 \mathrm{~K}$ ). Only one-quarter of the nanopore domain (in a square-packed

CNT array, $D=50 \mathrm{~nm}, p=2$ ) is considered. (d) Two-dimensional representation of wicking length $\left(L_{w}\right)$ across the CNT array with input heat flux $q^{\prime \prime}$ on the evaporator side of the vapor chamber. 
Figure 3. (a) The thermal resistance network model is illustrated for hexagonally-packed CNTs on a substrate. $R_{1}$ and $R_{2}$ are the conduction resistances in the liquid in the nanopore from the CNT surface and the substrate, respectively, while $R_{3}$ is the evaporative resistance of the water meniscus. $T_{\text {wall }}, T_{\text {vap }}$ are the substrate and vapor temperatures, respectively. $h_{m}$ represents the meniscus level in the nanopore. (b) Path for heat transfer from substrate to vapor.

Figure 4. Variation of (a) $\Delta P_{c a p}$ and (b) permeability with $\Phi$ for hexagonal- and square-packed posts. Variation of wicking length with CNT density for various diameters for (c) hexagonal, and (d) square packing, where the peaks indicate the optimum CNT density needed for maximizing the wicking length.

Figure 5. Variation of wick resistance with $\Phi$ for (a) hexagonal- and (b) square-packed posts.

Figure 6. Illustration of CNT-enhanced sintered copper particle wick (not to scale, 2D representations of the 3D calculations shown here): (a) particles with no enhancement, (b) particles with non-wicking CNTs, and (c) particles with completely wicking CNTs. Cases (b) and (c) represent the extreme scenarios for the wicking ability of the CNT forest.

Figure 7. The (a) thermal resistance network and (b) the path for heat transfer from a nanostructure-enhanced microstructure wick, respectively.

Figure 8. Thermal resistance of CNT-enhanced sintered particle wick obtained from the thermal resistance network (Figure 7(d, e)) plotted for varying CNT number density. The liquid level in the wick pore is varied from $h / r_{p}=0.6$ to 1.4 . Resistance values for the three cases shown in Figure 7(a, b, c) are plotted for comparison. 
Table 1. Wick porosity, pitch and base area of unit cell in terms of CNT number density (per $\mathrm{cm}^{2}$ ) and diameter $(D)$.

\begin{tabular}{|l|l|l|}
\hline Parameter & Hexagonal Packing & Square Packing \\
\hline Wick porosity $(\varepsilon)$ & $1-\frac{\pi}{2 \sqrt{3} p^{2}}$ & $1-\frac{\pi}{4 p^{2}}$ \\
\hline Pitch (p) & $\frac{2}{D} \sqrt{\frac{10^{-4}}{\sqrt{3} \phi}}$ & $\frac{1}{D} \sqrt{\frac{10^{-4}}{\phi}}$ \\
\hline Base area of unit cell $\left(A_{b}\right)$ & $\frac{\sqrt{3}}{4} p^{2} D^{2}$ & $p^{2} D^{2}$ \\
\hline
\end{tabular}




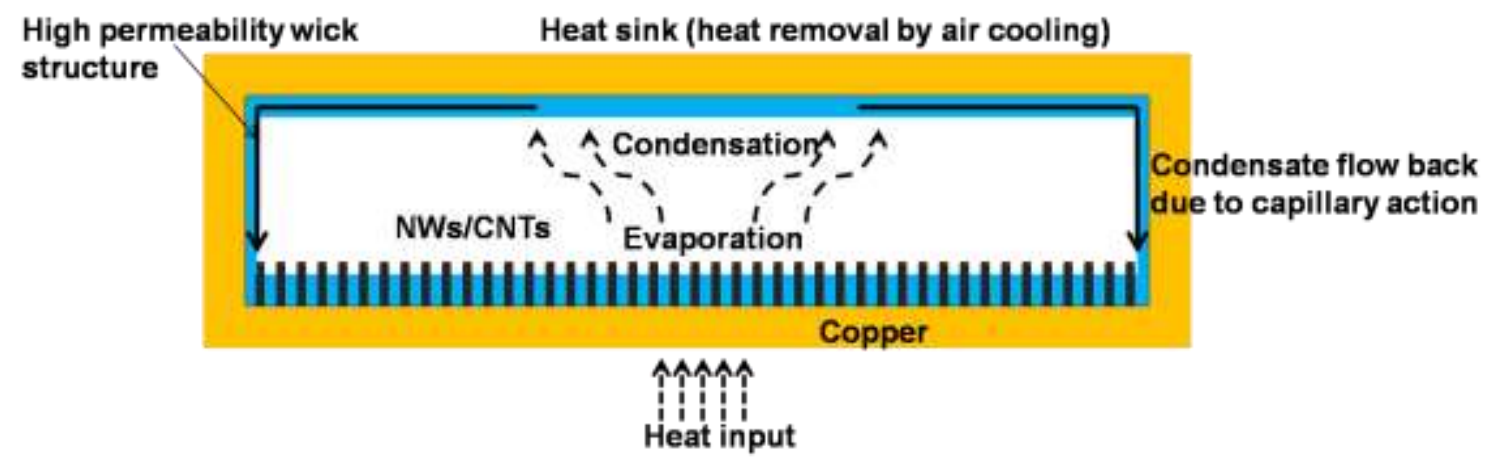

(a)

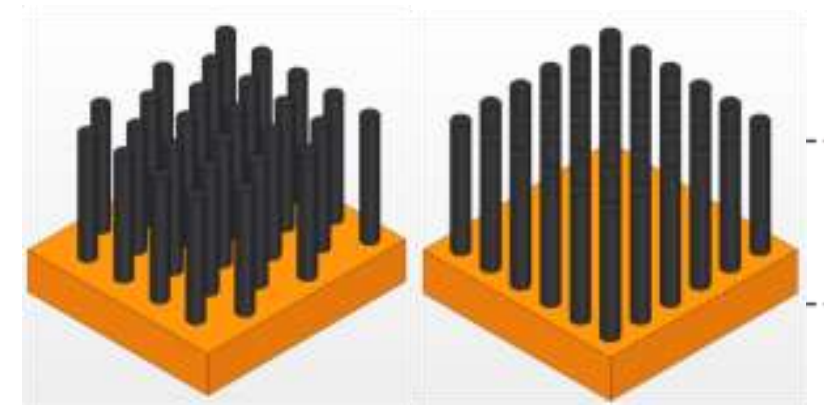

(b) (c)



(d)

Figure 1. (a) Schematic diagram of the operation of a vapor chamber heat spreader. CNTs/NWs are shown in the evaporator region. CNTs/NWs are shown as vertical posts in (b) hexagonal and (c) square packed arrangements on a copper substrate. (d) Plan view of square-packed posts with $D$ being the post diameter and $p$ the non-dimensional pitch. 


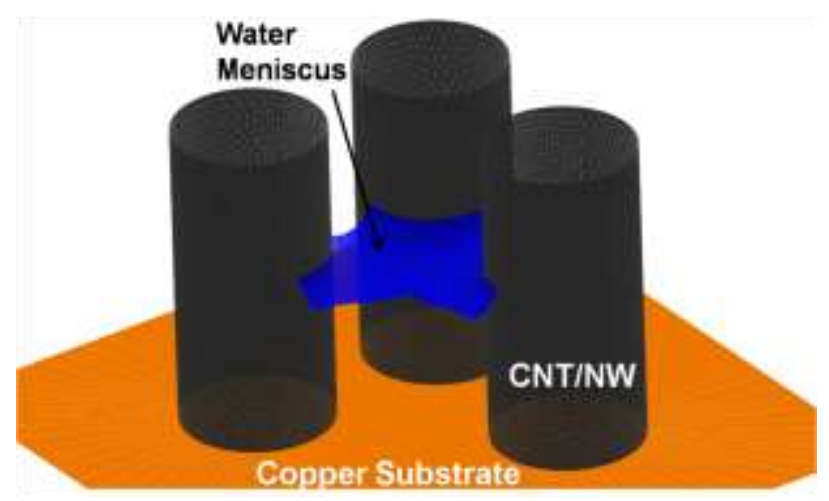

(a)

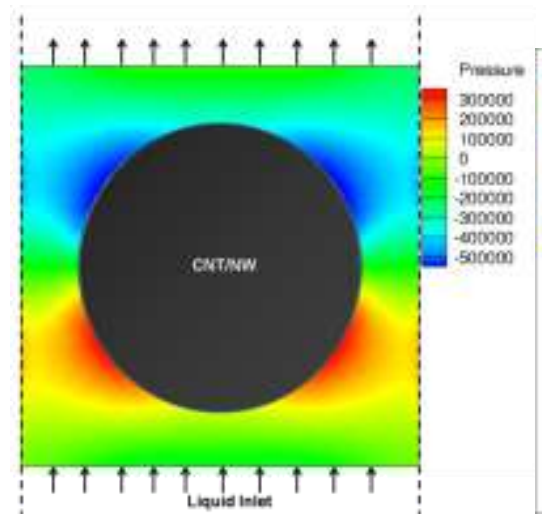

(b)

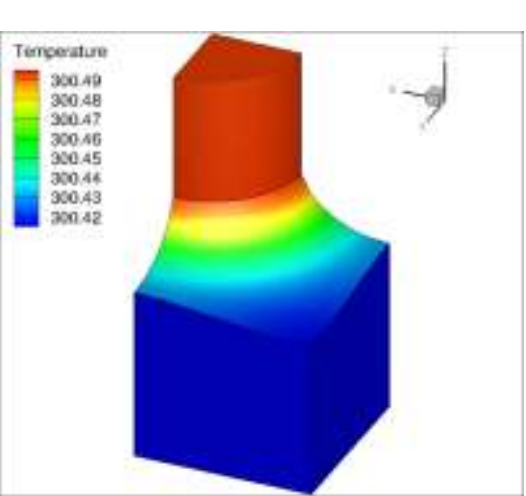

(c)

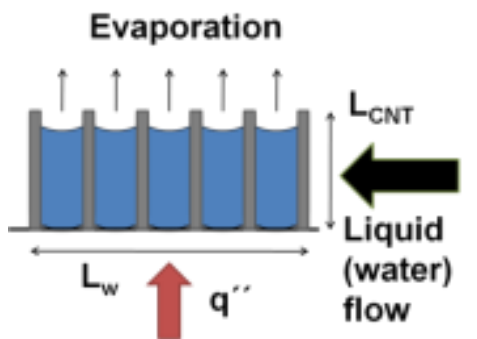

(d)

Figure 2. (a) The shape of the water meniscus in the nanopore formed between square-packed CNTs. The meniscus surface is puckered, exhibiting anticlastic curvature in the narrower spaces between two adjacent posts, while the surface is synclastic in the central pore region. (b) Pressure contours $(\mathrm{Pa})$ are shown in the liquid flow region around a square-packed post $(D=100$ $\mathrm{nm}, p=1.4$ ). The permeability of the nanowick is computed using Darcy's flow model for porous media. (c) Temperature contours $(\mathrm{K})$ during evaporation of water from the meniscus formed in a nanopore (square-packed posts) under saturated vapor conditions (wall superheat $=$ $2.5 \mathrm{~K}, \theta=15^{\circ}, T_{\text {vap }}=298 \mathrm{~K}$ ). Only one-quarter of the nanopore domain (in a square-packed CNT array, $D=50 \mathrm{~nm}, p=2$ ) is considered. (d) Two-dimensional representation of wicking length $\left(L_{w}\right)$ across the CNT array with input heat flux $q^{\prime \prime}$ on the evaporator side of the vapor chamber. 


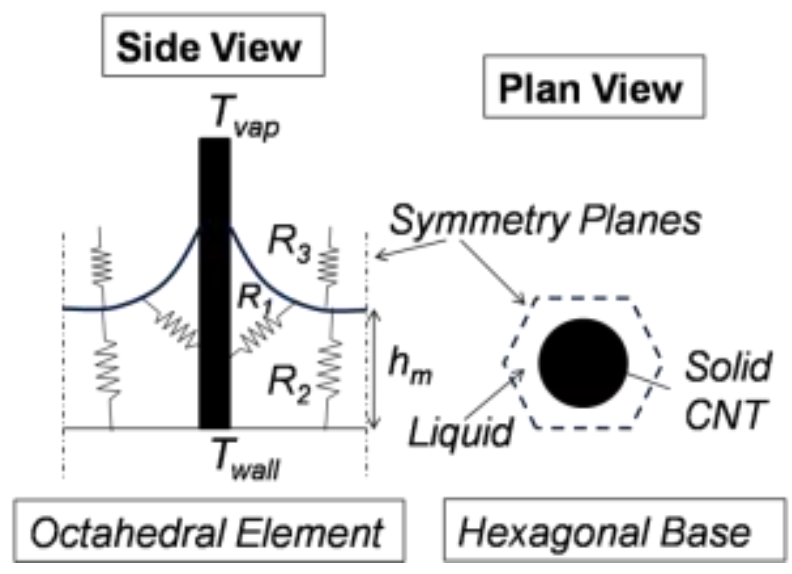

(a)

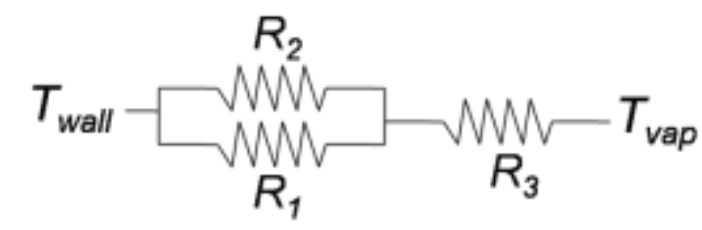

(b)

Figure 3. (a) The thermal resistance network model is illustrated for hexagonally-packed CNTs on a substrate. $R_{1}$ and $R_{2}$ are the conduction resistances in the liquid in the nanopore from the CNT surface and the substrate, respectively, while $R_{3}$ is the evaporative resistance of the water meniscus. $T_{\text {wall }}, T_{\text {vap }}$ are the substrate and vapor temperatures, respectively. $h_{m}$ represents the meniscus level in the nanopore. (b) Path for heat transfer from substrate to vapor. 


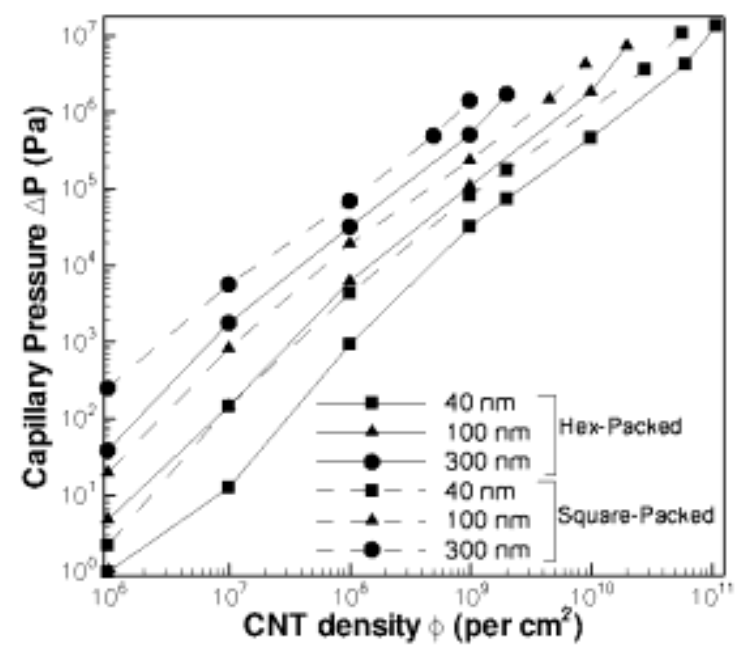

(a)

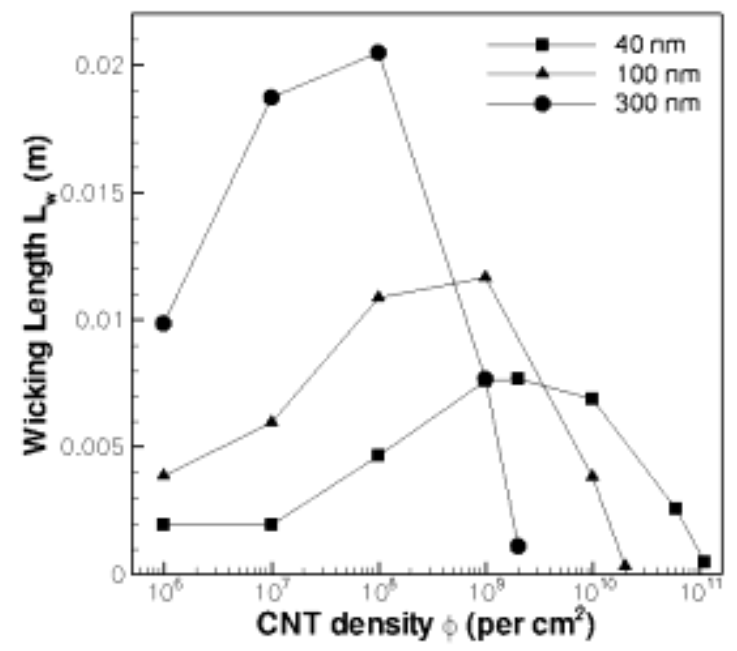

(c)



(b)



(d)

Figure 4. Variation of (a) $\Delta P_{c a p}$ and (b) permeability with $\Phi$ for hexagonal- and square-packed posts. Variation of wicking length with CNT density for various diameters for (c) hexagonal, and (d) square packing, where the peaks indicate the optimum CNT density needed for maximizing the wicking length. 


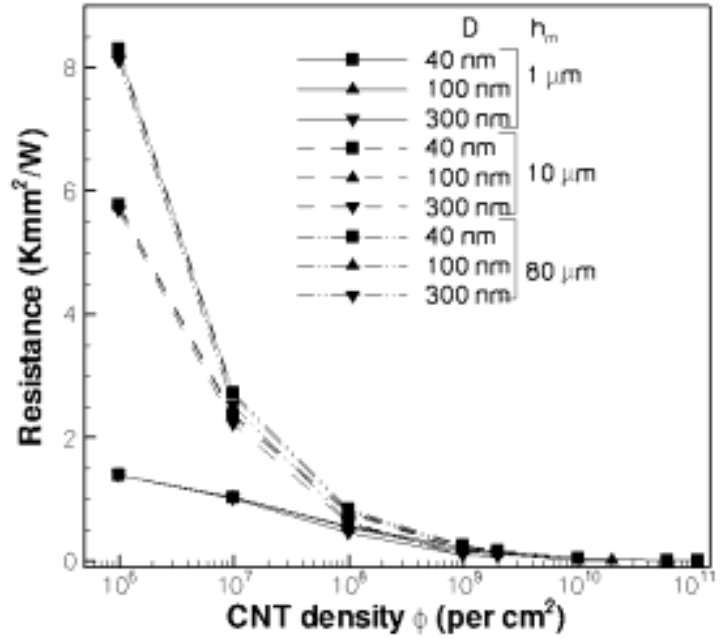

(a)



(b)

Figure 5. Variation of wick resistance with $\Phi$ for (a) hexagonal- and (b) square-packed posts. 




Figure 6. Illustration of CNT-enhanced sintered copper particle wick (not to scale, 2D representations of the 3D calculations shown here): (a) particles with no enhancement, (b) particles with non-wicking CNTs, and (c) particles with completely wicking CNTs. Cases (b) and (c) represent the extreme scenarios for the wicking ability of the CNT forest. 




(a)

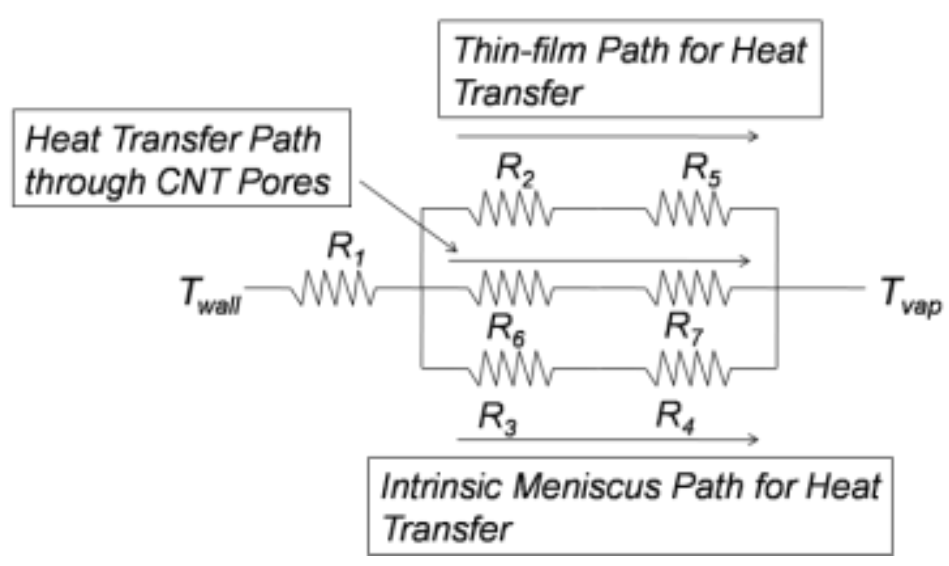

(b)

Figure 7. The (a) thermal resistance network and (b) the path for heat transfer from a nanostructure-enhanced microstructure wick, respectively. 


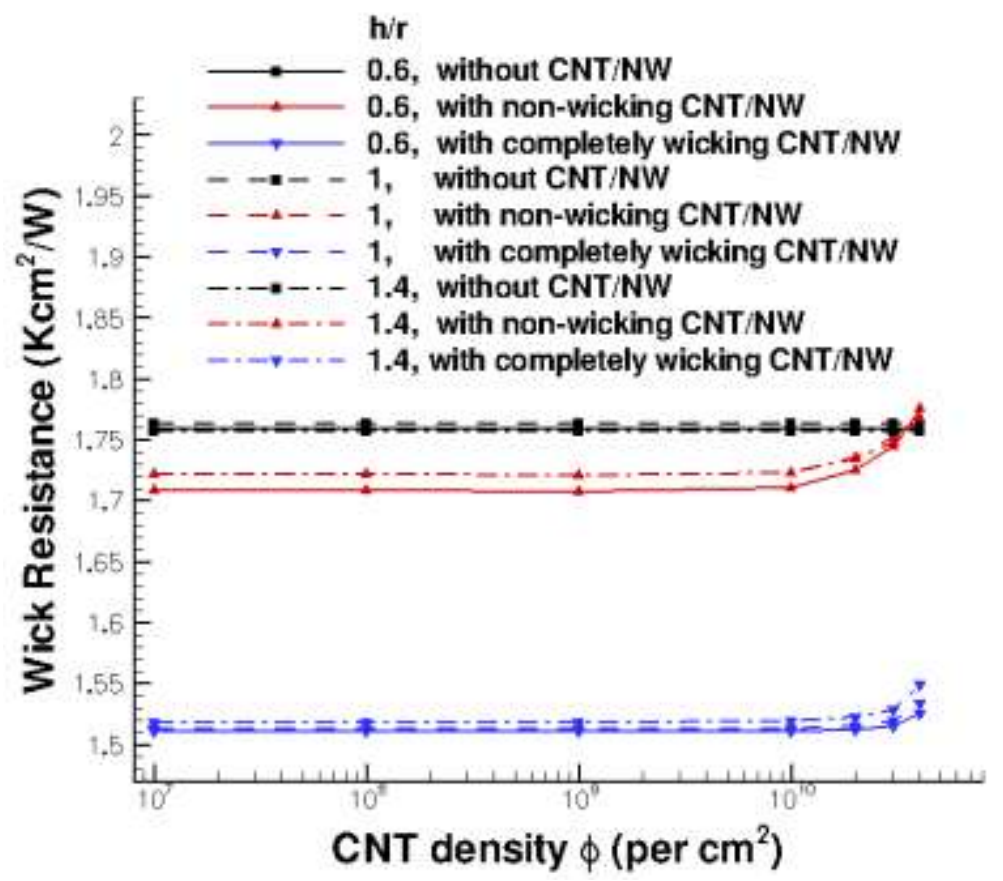

Figure 8. Thermal resistance of CNT-enhanced sintered particle wick obtained from the thermal resistance network (Figure 7(d, e)) plotted for varying CNT number density. The liquid level in the wick pore is varied from $h / r_{p}=0.6$ to 1.4 . Resistance values for the three cases shown in Figure $6(a, b, c)$ are plotted for comparison. 